\title{
LES PERFORATIONS PAR TRAUMATISME INTERNE DE L'CESOPHAGE
}

\author{
B. HAMMAMI, M. MNEJJA, Am. CHAKROUN, I. ACHOUR, \\ Ad. CHAKROUN, I. CHARFEDDINE, A. GHORBEL \\ SERVICE ORL ET CHIRURGIE CERVICO-FACIALE \\ CHU HABIB BOURGUIBA SFAX TUNISIE
}

\begin{abstract}
RESUME
Introduction : Les perforations traumatiques de l'œsophage sont rares mais graves mettant souvent en jeu le pronostic vital. Le but de ce travail est de rappeler les difficultés thérapeutiques des perforations traumatiques de l'œsophage.

Malades et méthodes : Il s'agit d'une étude rétrospective à propos de 7 malades suivis au CHU Habib Bourguiba pour perforation traumatique de l'œsophage. Nous avons étudié les étiologies des perforations ainsi que la prise en charge thérapeutique (médicale, chirurgicale) et le délai de son instauration. L'évolution a été évaluée après la fin du traitement et jugé bonne lors de l'obtention d'un rétablissement de la continuité de la filière digestive.

L'âge moyen des malades était de 24 ans. Deux malades avaient des antécédents à type de mégaoesophage idiopathique et sténose caustique. Le traumatisme était par corps étranger dans 2 cas, lors d'une extraction de corps étranger dans 3 cas et lors d'une endoscopie traumatique dans 2 cas.

Résultats : Le traitement a consisté en un traitement médical isolé dans 1 cas et associé à la chirurgie pour le reste des malades. Le traitement chirurgical était conservateur dans 2 cas et à type d'exclusion- remplacement œsophagien dans 4 cas. L'évolution post opératoire était favorable dans tous les cas.

Conclusion : Les perforations œsophagiennes peuvent mettre en jeu le pronostic vital par les complications graves qu'elles engendrent. Leur prise en charge est une urgence médico-chirurgicale. Le traitement médical doit être instauré dans tous les cas dès la suspicion du diagnostic. La chirurgie est indiquée d'emblée lors de complications ou durant la surveillance en l'absence d'amélioration sous traitement médical. La majorité des auteurs optent pour un traitement conservateur de l'œsophage surtout si le diagnostic est fait dans les 48 premières heures. La chirurgie non conservatrice est gardée pour les échecs du traitement conservateur.
\end{abstract}

Mots clés : Perforation, œsophage, corps étranger, endoscopie, chirurgie, pronostic.

Introduction: Esophagus traumatic perforations still carries high morbidity and mortality rates. There is no consensus regarding the appropriate management of this life-threatening condition.

The aim of this study is to analyse treatment of traumatic esophageal perforations.

Patients and methods: This retrospective study was made with seven patients treated for traumatic perforation of the esoephagus at the ENT department of the Habib Bourguiba Hospital. All patients were men. The mean age was 24 years. One patient has a history of an idiopathic megaesophagus and another have a history of a caustic stenosis.

The traumatism was by foreign body in 2 cases, at the time of an extraction of foreign body in 3 cases and at the time of a traumatic endoscopy in 2 cases.

Results: The treatment consisted on an isolated medical treatment in 1 case and associated to the surgery for the 6 other patients. The surgical treatment was conservative in 2 cases. Esophagus exclusion with replacement was made in 4 cases. After treatment, evolution was good for all patients.

Conclusion: Esophageal perforations can cause death by some serious complications. The treatment is a medico-surgical urgency. The medical treatment must be founded in all the cases as of the suspicion of the diagnosis. The surgery is indicated for complications or during the monitoring in the absence of improvement under medical treatment. The majority of the authors choose conservative treatment especially if the diagnosis is made in the 48 first hours. The radical surgery is kept for the failures of the conservative treatment.

Key words: Perforation, esophagus, foreign body, endoscopy, surgery, prognosis.

\section{INITRODUCTION}

La perforation traumatique de l'œsophage est une pathologie rare. Elle met en jeu le pronostic vital par la communication engendrée entre la lumière oesophagien- ne, milieu hyperseptique, et l'espace cellulo-graisseux médiastinal et/ou pleural, hautement vulnérable. Malgré les progrès des moyens diagnostiques et de réanimation, cette pathologie reste accompagnée d'une lourde 
morbidité et mortalité.

A travers la littérature et une série de 7 malades, atteints d'une perforation traumatique de l'œsophage, nous rappelons les difficultés thérapeutiques que posent les perforations traumatiques de l'œsophage.

\section{PATIENTS ET METHODES}

II s'agit d'une étude rétrospective à propos de 7 malades atteints de perforations traumatiques de l'œsophage, suivis et traités aux services ORL, chirurgie générale, chirurgie cardio-vasculaire et thoracique et de réanimation du CHU Habib Bourguiba de Sfax. L'étude a été réalisée sur une période de 11 ans (1987-1997). Les perforations tumorales ainsi que les perforations peropératoires ou par traumatisme externe ont été exclues de l'étude.

Nous avons étudié les étiologies des perforations ainsi que la prise en charge thérapeutique (médicale, chirurgicale) et le délai de son instauration. L'évolution a été évaluée après la fin du traitement et jugée bonne lors de l'obtention d'un rétablissement de la continuité de la filière digestive.

II s'agit de 7 malades de sexe masculin. L'âge moyen était de 24 ans avec des extrêmes de 6 et 63 ans. Deux patients avaient des antécédents pathologiques. II s'agissait d'un mégaoesophage idiopathique dans un cas et d'une sténose caustique de l'œsophage dans l'autre. Les étiologies des perforations étaient dominées par l'oesophagoscopie lors d'une extraction d'un corps étranger (tableau I).

\begin{tabular}{|l|c|c|}
\hline \multicolumn{1}{|c|}{ Etiologie } & Nombre & $\mathbf{\%}$ \\
\hline Extraction de CE & 3 & 43 \\
Endoscopie traumatique & 2 & 28,5 \\
CE perforant (fig.1) & 2 & 28,5 \\
\hline
\end{tabular}

CE : corps étranger

Tableau I : Etiologies des perforationsde l'oesophage

Le siège de la perforation était thoracique dans 5 cas et cervical dans 2 cas répartis selon l'étiologie dans le tableau II.

\begin{tabular}{|l|c|c|}
\hline \multicolumn{1}{|c|}{ Etiologie } & Perforation cervicale & Perforation thoracique \\
\hline Extraction d'un CE & 0 & 3 \\
Endoscopie traumatique & 0 & 2 \\
Corps étranger perforant & 2 & 0 \\
\hline
\end{tabular}

CE : corps étranger

Tableau II : Siège et étiologie de la perforation

Les signes d'appel de la perforation étaient dominés par la douleur cervicothoracique dans tous les cas. Une fièvre avec température entre 38 et $39^{\circ} 8$ a été notée dans 5 cas. Les signes digestifs (dysphagie, hypersialorrhée, vomissement) étaient présents dans 3 cas et les signes de détresse respiratoire dans 4 cas.
Le délai entre le traumatisme et l'apparition de ces signes était en moyenne de 10 heures avec des extrêmes de 3 et 48 heures. II a été de 4 et 48 heures en cas de corps étranger perforant et de 3 heures en moyenne pour les autres circonstances.

L'endoscopie oesophagienne a été réalisée chez tous les patients, dans un but diagnostique dans tous les cas et en plus thérapeutique dans 5 cas.

La radiographie du thorax a été réalisée de façon systématique dans tous les cas. Elle a montré un corps étranger dans 4 cas et un hydropneumothorax dans 2 cas (fig.1). Le corps étranger était un crochet de prothèse dentaire dans 2 cas, une pièce de monnaie dans 1 cas et un fil de fer enroulé dans 1 cas (fig.2).

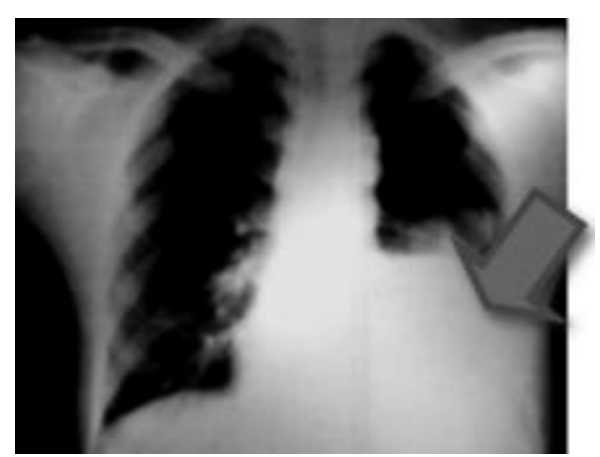

Fig.1 : Radiographie du thorax : Hydropneumothorax gauche (observation 2)

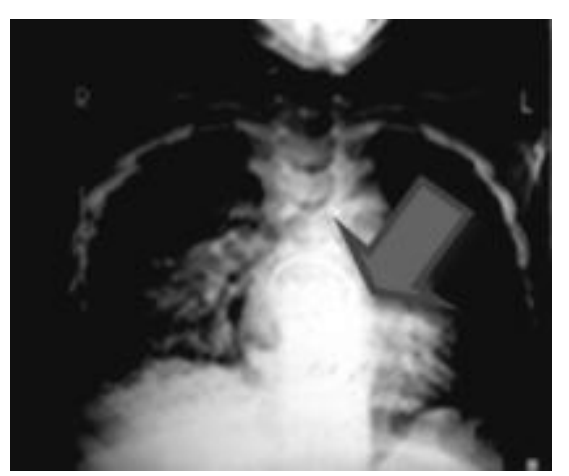

Fig.2 : Radiographie du thorax :

Corps étranger tranchant (fil d'acier enroulé) en regard de D7-D9 (observation 4)

Le diagnostic de perforation oesophagienne a été confirmé dans tous les cas par l'imagerie. Une opacification aux hydrosolubles a été réalisée dans 6 cas (fig.3) et la tomodensitométrie a été effectuée dans 3 cas. Elle a montré en plus des lésions médiastinales et / ou pulmonaires (tableau III) (fig.4). 


\begin{tabular}{|l|c|}
\hline \multicolumn{1}{|c|}{ Lésion } & Nombre de malades \\
\hline Médiastinite & 1 \\
Pneumomédiastin & 2 \\
Abcès pulmonaire & 2 \\
Pleurésie & 1 \\
Hydropneumothorax & 1 \\
Corps étranger & 1 \\
Perforation oesophagienne & 1 \\
\hline
\end{tabular}

Tableau III : Répartition des lésions diagnostiquées à la TDM

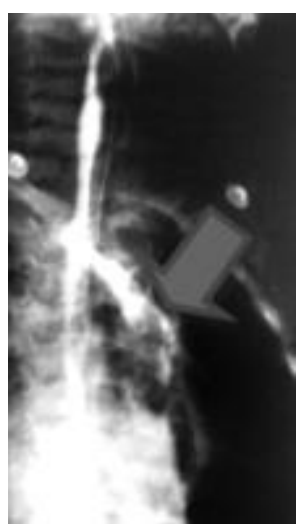

Fig.3 : Transit œsophagien à la gastrografine: Perforation œsophagienne à la jonction tiers supérieur - tiers moyen (observation 3)

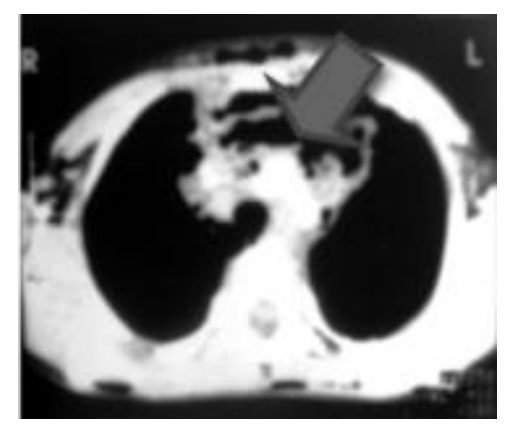

Fig.4 : TDM thoracique : médiastinite avec pneumomédiastin (observation 3)

\section{RESULTATS}

La prise en charge de ces malades était médico-chirurgicale (tableau IV). Le traitement médical a consisté en la mise en route d'une alimentation entérale par sonde nasogastrique avec antibiothérapie à large spectre. Le délai préthérapeutique (intervalle libre entre la perforation et l'intervention) était en moyenne de 4,16 jours avec des extrêmes de 2 et 5 jours.

\begin{tabular}{|c|c|c|c|c|c|}
\hline Obs. & $\begin{array}{l}\text { Age } \\
\text { (ans) }\end{array}$ & Antécédents & C.E. & Complications & Traitement \\
\hline 1 & 30 & - & $\begin{array}{c}\text { Crochet } \\
\text { métallique }\end{array}$ & - & $\begin{array}{l}\text { Echec de la } \\
\text { suture, apposition } \\
\text { du chef du muscle } \\
\text { SCM }\end{array}$ \\
\hline 2 & 40 & $\begin{array}{c}\text { Méga- } \\
\text { œsophage } \\
\text { idiopathique }\end{array}$ & $\begin{array}{c}\text { Tentative de } \\
\text { dilatation }\end{array}$ & $\begin{array}{l}\text { Hydropneumo- } \\
\text { thorax }\end{array}$ & $\begin{array}{l}\text { Exclusion } \\
\text { œsophage- } \\
\text { remplacement }\end{array}$ \\
\hline 3 & 6 & - & $\begin{array}{l}\text { Pièce de } \\
\text { monnaie }\end{array}$ & $\begin{array}{l}\text { Médiastinite } \\
\text { Pneumo- } \\
\text { médiastin }\end{array}$ & $\begin{array}{l}\text { Exclusion } \\
\text { remplacement de } \\
\text { l'œsophage }\end{array}$ \\
\hline 4 & 8 & épilepsie & $\begin{array}{c}\text { Fil d'acier } \\
\text { enroulé }\end{array}$ & $\begin{array}{c}\text { Abcés } \\
\text { pulmonaire } \\
\text { droit }\end{array}$ & $\begin{array}{l}\text { Exclusion } \\
\text { remplacement de } \\
\text { l'œsophage }\end{array}$ \\
\hline 5 & 8 & $\begin{array}{l}\text { Sténose } \\
\text { caustique }\end{array}$ & $\begin{array}{c}\text { Tentative de } \\
\text { dilatation }\end{array}$ & $\begin{array}{l}\text { Pleurésie } \\
\text { bilatérale }\end{array}$ & $\begin{array}{l}\text { Drainage pleural } \\
\text { Exclusion } \\
\text { remplacement de } \\
\text { l'œsophage }\end{array}$ \\
\hline 6 & 15 & - & $\begin{array}{l}\text { Oesophago- } \\
\text { scopie pour } \\
\text { CE }\end{array}$ & pleurésie & $\begin{array}{l}\text { Drainage pleural } \\
\text { Simple suture de } \\
\text { perforation }\end{array}$ \\
\hline 7 & 63 & - & $\begin{array}{c}\text { Extraction } \\
\text { De crochet } \\
\text { de dentier }\end{array}$ & - & $\begin{array}{l}\text { Ttt médical } \\
\text { Repos du tube } \\
\text { digestif }\end{array}$ \\
\hline
\end{tabular}

Tableau IV : Tableau récapitulatif des observations

Pour les perforations cervicales, le traitement était exclusivement médical dans un cas. II s'agissait d'une perforation punctiforme diagnostiquée lors de l'extraction d'un corps étranger. Dans le deuxième cas, le traitement était médico-chirurgical. La chirurgie était conservatrice instaurée dès le diagnostic positif vu la taille de la perforation qui était considérable. Elle a consisté en une suture de la perforation avec recouvrement par le chef sternal du muscle sternocleidomastoidien .

Pour les 5 perforations thoraciques, tous les malades ont été opérés par voie de thoracotomie.

- Il s'agissait dans 1 cas, d'une perforation de $2 \mathrm{~cm}$ de longueur pour laquelle le malade a eu une suture de proche en proche. Le délai entre la perforation et la chirurgie était de $24 \mathrm{H}$. L'alimentation a été autorisée au 8éme jour après un transit oesophagien normal.

- Pour les 4 autres malades, le délai moyen entre la survenue de la perforation et la chirurgie était de 3 jours. Une double exclusion oesophagienne avec oesophagectomie subtotale a été réalisée. Le rétablissement de la continuité oesophagienne était différé dans tous ces cas. Le délai moyen entre les 2 actes chirurgicaux était de 105 jours avec des extrêmes de 59 et 136 jours. L'alimentation par jéjunostomie a été entamée en moyenne 14 jours post opératoire. La reprise alimentaire par voie orale a été autorisée 58 jours post opératoire après obtention d'un transit digestif normal.

L'évolution était favorable dans tous les cas. Les malades 
étaient vus à des contrôles réguliers avec un recul à 3 mois après la deuxième chirurgie. Le rétablissement de la continuité des voies digestives hautes a été vérifié par un transit œsophagien (fig.5).

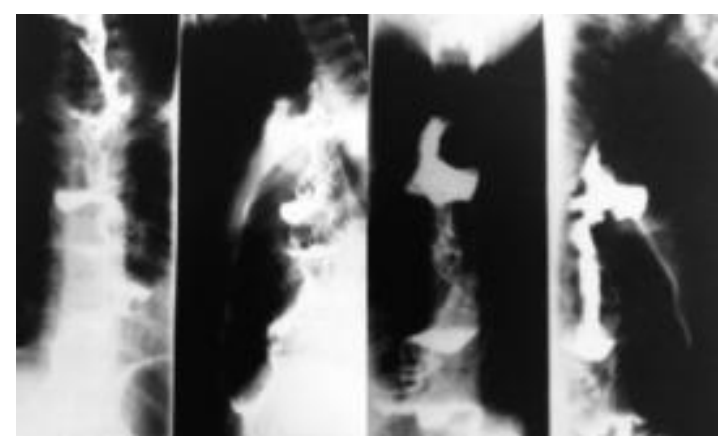

Fig.5 : Transit œsophagien à 5 mois et à 12 mois post opératoire : Absence de fuite de produit de contraste (observation3)

\section{DISCUSSION}

La perforation traumatique de l'œsophage est rare et grave. Elle est responsable d'une mortalité de 0 à $45 \%$ selon le siège de la perforation, le terrain et la cause $(4$, $5,7)$. La plus grande série retrouvée dans la littérature comporte 64 cas de perforation traumatique de l'œsophage sur 20 ans (3).

Les étiologies sont dominées par les manœuvres instrumentales : endoscopie digestive (diagnostic, extraction de corps étranger ou pose d'une prothèse duodénale), tentative d'intubation, traitement endoscopique de varices œsophagiennes ou encore une échographie transoesophagienne $(4,5,9)$. Les dilatations sur œsophage pathologique exposent également au risque de perforation traumatique instrumentale. Le corps étranger lui-même peut également perforer l'œsophage $(6,7)$. D'autres étiologies sont beaucoup plus rares et se voient exceptionnellement dans la littérature à savoir l'ostéosynthèse rachidienne et le lavage gastrique (5).

Le siège de la perforation est prédominant au niveau thoracique ou à la jonction cervico-thoracique (tableau V).

\begin{tabular}{|l|c|c|c|}
\hline & Cervicale & thoracique & Total \\
\hline Richardson [3] & 13 & 51 & 64 \\
Cheynel [5] & 7 & 33 & 40 \\
Notre série & 2 & 5 & 7 \\
\hline
\end{tabular}

Tableau V : Siège de la perforation œsophagienne

Le diagnostic positif est souvent évoqué devant le contexte. La confirmation est obtenue par l'imagerie qui objective la rupture et son siège.

La communication crée par la perforation de l'œsophage entre un milieu septique (lumière œsophagienne) et le médiastin (aseptique et vulnérable) fait que le traitement est urgent et son début est indiqué dès la suspicion de la lésion.

La prise en charge thérapeutique est médico-chirurgicale. Elle doit être la plus précoce possible.

\section{- Traitement médical}

Le traitement médical constitue le premier volet thérapeutique. II est instauré de façon systématique chez tous les malades dès que le diagnostic est évoqué. II consiste en la mise au repos du tube digestif avec mise d'une sonde nasogastrique associée à une antibiothérapie à large spectre. L'alimentation sera assurée par voie entérale ou parentérale. Ce traitement peut être suffisant à lui seul et ce dans les perforations de petites taille, de diagnostic précoce, le plus souvent cervicale et en dehors de toute complication $(1,4,5,6,9)$.

Le traitement médical ne doit pas retarder la prise en charge chirurgicale. En effet, dès la constatation d'une évolution défavorable avec persistance ou apparition de signes thoraciques de complication, il faut passer à la chirurgie. (4).

Dans notre série, un malade a été traité uniquement médicalement avec une bonne évolution.

Une surveillance stricte clinique et radiologique doit être réalisée afin de détecter les cas ayant mal évolués et les complications. Le traitement des complications relève aussi d'un traitement médical de réanimation en premier temps.

\section{- Traitement chirurgical}

Le traitement chirurgical est indiqué en cas d'échec du traitement médical. II est aussi indiqué en association avec le traitement médical en cas de formes compliquées d'emblée et en cas de perforations larges (1, 9, 10). Ce traitement doit prendre en considération le siège et la taille de la perforation ainsi que le temps entre la survenue de la perforation et le diagnostic. Cette chirurgie vise la perforation elle-même mais aussi les différentes complications. Elle peut être conservatrice ou non vis-à-vis de l'œsophage.

La voie d'abord cervicale permet souvent d'atteindre les perforations jusqu'au niveau de la 4ème vertèbre thoracique (10). Le recours à une voie d'abord thoracique est l'apanage des perforations thoraciques basses et des complications médiastino-pulmonaires.

\section{$>$ Chirurgie conservatrice}

Pour le traitement des perforations oesophagiennes, la tendance de la majorité des auteurs est d'être le plus conservateur possible $(3,4,5)$. Ce traitement peut être à type de drainage en regard de la fuite oesophagienne ou d'une simple suture de proche en proche de la perforation $(4,5,10)$.

Le drainage au contact de la perforation est justifié au niveau cervical car il évite une fuite vers le médiastin. Pour les perforations de l'œsophage thoracique, il peut constituer un premier temps de traitement en cas de pleurésie ou de collection médiastinale (10). 
La tendance actuelle dans la littérature est d'élargir les indications à la suture des lésions de l'œsophage thoracique, autrefois préconisé pour l'œsophage cervical, même en cas de retard de diagnostic $(2,8)$. Pour Cheynel, ce traitement doit être plutôt indiqué pour les perforations avant $36 \mathrm{~h}$ et en dehors de toute complication quelque soit son siège (5). La suture oesophagienne peut être protégée par un plan musculaire de voisinage. Ce traitement conservateur permet de réduire la durée d'hospitalisation et la morbidité $(2,5,8,9)$. Rubikas n'a pas trouvé de différence significative dans la durée d'hospitalisation entre le drainage aspiration et la suture des lésions (10).

Dans notre série, un traitement chirurgical conservateur a été réalisé dans deux cas. II s'agissait d'une perforation cervicale dans un cas pour lequel nous avons pratiqué une apposition d'un chef du muscle sternocleidomastoisien et un cas de perforation thoracique sur lequel nous avons réalisé une simple suture. L'évolution était favorable dans les 2 cas. La durée d'hospitalisation dans ces deux cas était de 10 et 15 jours.

\section{$>$ Chirurgie non conservatrice}

Le traitement non conservateur consiste en l'exclusion oesophagienne, oesophagectomie et rétablissement de la continuité du tube digestif par remplacement oesophagien dans le même temps ou dans un deuxième temps. Le remplacement oesophagien peut se faire par tubulisation gastrique, implant jéjunal ou coloplastie. La durée d'hospitalisation et la morbidité est plus élevée dans ce type de chirurgie.

L'oesophagectomie permet un débridement efficace du médiastin en cas de médiastinite avérée. Lors de l'oesophagectomie, le maximum d'œsophage doit être conservé pour faciliter le rétablissement de la continuité (5).
Le traitement non conservateur est indiqué surtout pour les perforations thoraciques. L'échec du traitement conservateur, les perforations larges non suturables ainsi que le diagnostic de complication médiastinale d'emblée en constituent les indications les plus courantes.

Le pronostic de ces perforations est souvent réservé. Le taux de mortalité est de 0 à $45 \%(4,5,7)$. Pour Cheynel, le taux global de mortalité était de $17 \%$. II a été de $21 \%$ après rupture spontanée et $19 \%$ après perforation iatrogène. La mortalité était de $14 \%$ pour les lésions cervicales et $18 \%$ pour les lésions thoraciques sans différence significative (5). La cause de décès est souvent une défaillance multiviscérale imputable à une médiastinite.

\section{CONCLUSION}

La perforation traumatique de l'œsophage est une pathologie rare entachée d'une lourde mortalité. Le diagnostic est souvent évoqué devant le contexte. Elle doit être suspectée devant toute anomalie au cours ou au décours d'une endoscopie même diagnostique. Le diagnostic positif est assuré par un transit aux hydrosolubles ou par une tomodensitométrie qui permet en plus de détecter des complications associées.

Le traitement médical est systématique et souvent d'appoint associé au traitement chirurgical. La suture est le traitement de référence. L'oesophagectomie est nécessaire en cas de lésion non suturable ou en cas de complication médiastinale. La prévention reste le meilleur moyen pour éviter une morbidité et une mortalité élevées.

\section{REFERENCES}

1.Poluri A, Singh B, Sperling $\mathrm{N}$ et al. Retropharyngeal abscess secondary to penetrating foreign bodies. J Craniomaxillofac Surg. 2000; 28:243-246.

2.Brichon PY, Couraud L, Velly JF et al. Les perforations et ruptures de l'œsophage, à propos de 35 cas. Ann Chir. 1990; 44:464-70.

3.David Richardson J. Management of oesophageal perforations : the value of aggressive surgical treatment. Am. J. Surg., 2005; 190 (2):161-165.

4.Jougon J, Delcambre F, MacBride T et al. La mortalité des perforations instrumentales de l'œsophage est élevée : expérience de 54 cas traités. Ann Chir. 2002; 127(1):26-31.

5.Cheynel N, Arnal E, Peschaud Fet al. Perforation et rupture de l'œsophage : prise en charge et pronostic.Ann Chir. 2003; 128(3):163-166.
6.Mathur NN, Kumar S, Bothra R. Intramural foreign body in oesophagus. Int. J. Pediatr Otorhinolaryngol. 2004; 68(6):837-839.

7.Campisi P, Stewart C, Forte V. Penetrating oesophageal injury by ingestion of a wire bristle. J. Pediatr. Surg.. 2005; 40(10):15-16.

8.Platel JP, Thomas P, Giudicelli R et al. Perforations et ruptures de l'œsophage : plaidoyer pour un traitement conservateur. Ann Chir 1997; 51:611-6.

9.Badoui R, Choufane S, Riboulot $\mathrm{M}$ et al. Perforation oesophagienne après échocardiographie transoesophagienne. Ann Fr Anesth Reanim. 1994; 13:850852

10.Rubikas R. Pharyngeal and oesophageal injuries. Injury. 2004; 35:371-378. 